\title{
Colored trees and noncommutative symmetric functions
}

\author{
Matt Szczesny \\ Department of Mathematics \\ Boston University, Boston MA, USA \\ szczesny@math.bu.edu
}

Submitted: Oct 16, 2009; Accepted: Mar 28, 2010; Published: Apr 5, 2010

\begin{abstract}
Let $\mathcal{C} \mathcal{R} \mathcal{F}_{S}$ denote the category of $S$-colored rooted forests, and $\mathrm{H}_{\mathcal{C R F}_{S}}$ denote its Ringel-Hall algebra as introduced in [6]. We construct a homomorphism from a $K_{0}^{+}\left(\mathcal{C} \mathcal{R} \mathcal{F}_{S}\right)$-graded version of the Hopf algebra of noncommutative symmetric functions to $\mathrm{H}_{\mathcal{C R F}_{S}}$. Dualizing, we obtain a homomorphism from the Connes-Kreimer Hopf algebra to a $K_{0}^{+}\left(\mathcal{C} \mathcal{R} \mathcal{F}_{S}\right)$-graded version of the algebra of quasisymmetric functions. This homomorphism is a refinement of one considered by W. Zhao in [9].
\end{abstract}

\section{Introduction}

In [6] categories $\mathcal{L R} \mathcal{F}, \mathcal{L F} \mathcal{G}$ of labeled rooted forests and labeled Feynman graphs where constructed, and were shown to possess many features in common with those of finitary abelian categories. In particular, one can define their Ringel-Hall algebras $\mathrm{H}_{\mathcal{L R F}}, \mathrm{H}_{\mathcal{L F \mathcal { G }}}$. If $\mathcal{C}$ is one of these categories, $\mathrm{H}_{\mathcal{C}}$ is the algebra of functions on isomorphism classes of $\mathcal{C}$, equipped with the convolution product

$$
f \star g(M):=\sum_{A \subset M} f(A) g(M / A),
$$

and the coproduct

$$
\Delta(f)(M, N):=f(M \oplus N),
$$

where $M \oplus N$ denotes disjoint union of forests/graphs. Together, the structures 1.1 and 1.2 assemble to form a co-commutative Hopf algebra, which was in [6] shown to be dual to the corresponding Connes-Kreimer Hopf algebra ([5], [2]). In [6], we also defined the Grothendieck groups $K_{0}(\mathcal{C})$ for $\mathcal{C}=\mathcal{L} \mathcal{R} \mathcal{F}, \mathcal{L F} \mathcal{G}$ and showed that $\mathrm{H}_{\mathcal{C}}$ is naturally graded by $K_{0}^{+}(\mathcal{C})$ - the effective cone inside $K_{0}(\mathcal{C})$.

From the point of view of Ringel-Hall algebras of finitary abelian categories, the characteristic functions of classes in $K_{0}^{+}$are interesting. If $\mathcal{A}$ is such a category, and $\alpha \in K_{0}^{+}(\mathcal{A})$, 
we may consider $\kappa_{\alpha}$ - the characteristic function of the locus of objects of class $\alpha$ inside $\operatorname{Iso}(\mathcal{A})$ (for a precise definition, see [4]). It is shown there that the $\kappa_{\alpha}$ satisfy

$$
\Delta\left(\kappa_{\alpha}\right)=\sum_{\substack{\alpha_{1}+\alpha_{2}=\alpha \\ \alpha_{1}, \alpha_{2} \in K_{0}^{+}(\mathcal{A})}} \kappa_{\alpha_{1}} \otimes \kappa_{\alpha_{2}} .
$$

In this note, we show that these identities hold also when $\mathcal{A}$ is replaced by the category $\mathcal{C R} \mathcal{F}$ of colored rooted forests. If $S$ is a set, and $\mathcal{C} \mathcal{R} \mathcal{F}_{S}$ denotes the category of rooted forests colored by $S$, we show that $K_{0}\left(\mathcal{C} \mathcal{R} \mathcal{F}_{S}\right)=\mathbb{Z}^{|S|}$, and if $\alpha \in K_{0}^{+}\left(\mathcal{C} \mathcal{R} \mathcal{F}_{S}\right)$, we may define

$$
\kappa_{\alpha}:=\sum_{\substack{A \in \operatorname{Iso}\left(\mathcal{C R} \mathcal{F}_{S}\right) \\[A]=\alpha}} \delta_{A}
$$

i.e. the sum of delta functions supported on isomorphism classes with K-class $\alpha$. We show that the $\kappa_{\alpha}$ satisfy the identity 1.3.

As an application, we construct a homomorphism to $\mathrm{H}_{\mathcal{C R F}_{S}}$ from a $K_{0}^{+}\left(\mathcal{C R}_{\mathcal{R}} \mathcal{F}_{S}\right)$-graded version of the Hopf algebra of non-commutative symmetric functions (see [3]). More precisely, let $\mathrm{NC}_{\mathcal{C R F}_{S}}$ denote the free associative algebra on generators $X_{\alpha}, \alpha \in K_{0}^{+}\left(\mathcal{C} \mathcal{R} \mathcal{F}_{S}\right)$, to which we assign degree $\alpha$. We may equip it with a coproduct determined by the requirement

$$
\Delta\left(X_{\alpha}\right):=\sum_{\substack{\alpha_{1}+\alpha_{2}=\alpha \\ \alpha_{1}, \alpha_{2} \in K_{0}^{+}\left(\mathcal{C R F}_{S}\right)}} X_{\alpha_{1}} \otimes X_{\alpha_{2}},
$$

with which it becomes a connected graded bialgebra, and hence a Hopf algebra. We may now define a homomorphism

$$
\begin{aligned}
\rho: \mathrm{NC}_{\mathcal{C R F}_{S}} & \rightarrow \mathrm{H}_{\mathcal{C R F}_{S}} \\
\rho\left(X_{\alpha}\right) & :=\kappa_{\alpha} .
\end{aligned}
$$

This is a refinement of a homomorphism originally considered in [9]. Taking the transpose of $\rho$, we obtain a homomorphism from the Connes-Kreimer Hopf algebra to a $K_{0}^{+}\left(\mathcal{C R}_{\mathcal{S}}\right)-$ graded version of the Hopf algebra of quasisymmetric functions.

Acknowledgements: I would like to thank Dirk Kreimer for many valuable conversations, and the referee for their helpful comments.

\section{Recollections on $\mathcal{C} \mathcal{R} \mathcal{F}_{S}$}

We briefly recall the definition and necessary properties of the category $\mathcal{C} \mathcal{R} \mathcal{F}_{S}$, and calculate its Grothendieck group. For details and proofs, see [6]. While [6] treats the case of uncolored trees, the extension of the results to the colored case is immediate. Please note that the notion of labeling in [6] and coloring used here are distinct. 


\subsection{The category $\mathcal{C} \mathcal{R} \mathcal{F}_{S}$}

We begin by reviewing some notions related to rooted trees. Let $S$ be a set. For a tree $T$, denote by $V(T), E(T)$ the vertex and edge sets of $T$ respectively.

Definition 2.1. $\quad$ 1. A rooted tree colored by $S$ is a tree $T$, with a distinguished vertex $r(T) \in V(T)$ called the root, and an map $l: V(T) \rightarrow S$. An isomorphism between two trees $T_{1}, T_{2}$ labeled by $S$ is a pair of bijections $f_{v}: V\left(T_{1}\right) \simeq V\left(T_{2}\right), f_{e}: E\left(T_{1}\right) \simeq$ $E\left(T_{2}\right)$ which preserve roots, colors, and all incidences - we often refer to this data simply by $f$. Denote by $R T(S)$ the set of all rooted trees labeled by $S$.

2. A rooted forest colored $S$ is either empty, or an ordered set $F=\left\{T_{1}, \ldots, T_{n}\right\}$ where $T_{i} \in R T(S)$. Two forests $F_{1}=\left\{T_{1}, \ldots, T_{n}\right\}$ and $F_{2}=\left\{T_{1}^{\prime}, \ldots, T_{m}^{\prime}\right\}$ are isomorphic if $m=n$ and there is a permutation $\sigma \in S_{n}$, together with isomorphisms $f_{i}: T_{i} \simeq T_{\sigma(i)}^{\prime}$.

3. An admissible cut of a labeled colored tree $T$ is a subset $C(T) \subset E(T)$ such that at most one member of $C(T)$ is encountered along any path joining a leaf to the root. Removing the edges in an admissible cut divides $T$ into a colored rooted forest $P_{C}(T)$ and a colored rooted tree $R_{C}(T)$, where the latter is the component containing the root. The empty and full cuts $C_{n u l l}, C_{\text {full }}$, where

$$
\left(P_{C_{\text {null }}}(T), R_{C_{\text {null }}}(T)\right)=(\emptyset, T) \text { and }\left(P_{C_{\text {full }}}(T), R_{C_{\text {full }}}(T)\right)=(T, \emptyset)
$$

respectively, are considered admissible.

4. An admissible cut on a colored forest $F=\left\{T_{1}, \ldots, T_{k}\right\}$ is a collection of cuts $C=$ $\left\{C_{1}, \ldots, C_{k}\right\}$, with $C_{i}$ an admissible cut on $T_{i}$. Let

$$
\begin{aligned}
R_{C}(F) & :=\left\{R_{C_{1}}\left(T_{1}\right), \ldots, R_{C_{k}}\left(T_{k}\right)\right\} \\
P_{C}(F) & :=P_{C_{1}}\left(T_{1}\right) \cup P_{C_{2}}\left(T_{2}\right) \cup \ldots \cup P_{C_{k}}\left(T_{k}\right)
\end{aligned}
$$

Example 2.1. Consider the labeled rooted forest consisting of a single tree $T$ colored by $S=\{a, b\}$ with root drawn at the top,

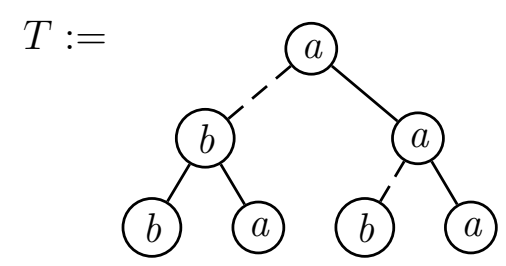

and the cut edges indicated with dashed lines. Then

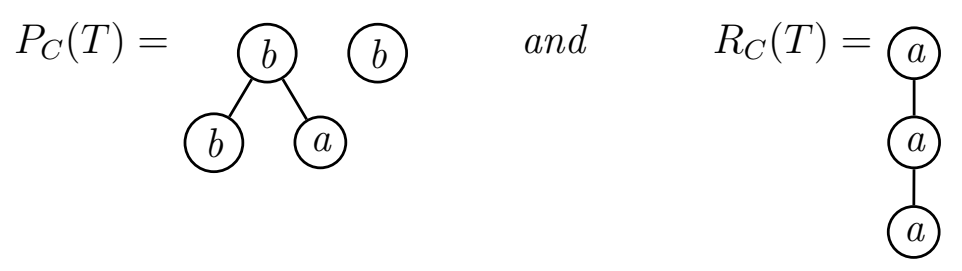


We are now ready to define the category $\mathcal{C} \mathcal{R} \mathcal{F}_{S}$, of rooted forests colored by $S$.

Definition 2.2. The category $\mathcal{C} \mathcal{R} \mathcal{F}_{S}$ is defined as follows:

$$
\begin{aligned}
\operatorname{Ob}\left(\mathcal{C} \mathcal{R} \mathcal{F}_{S}\right):= & \{\text { rooted forests } F \text { colored by } \mathrm{S}\} \\
\operatorname{Hom}\left(F_{1}, F_{2}\right):= & \left\{\left(C_{1}, C_{2}, f\right) \mid C_{i} \text { is an admissible cut of } F_{i},\right. \\
& \left.f: R_{C_{1}}\left(F_{1}\right) \cong P_{C_{2}}\left(F_{2}\right)\right\} .
\end{aligned}
$$

Note: For $F \in \mathcal{C R \mathcal { F }}_{S},\left(C_{\text {null }}, C_{\text {full }}, i d\right): F \rightarrow F$ is the identity morphism in $\operatorname{Hom}(F, F)$. We denote by Iso $\left(\mathcal{C} \mathcal{R} \mathcal{F}_{S}\right)$ the set of isomorphism classes of objects in $\mathcal{C} \mathcal{R} \mathcal{F}_{S}$.

Example: if
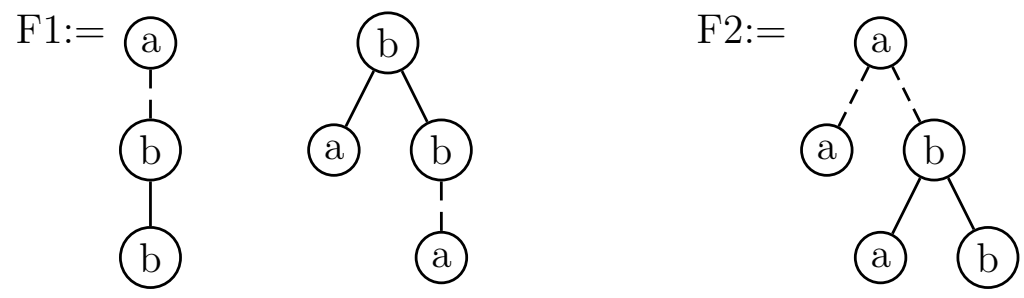

then we have a morphism $\left(C_{1}, C_{2}, f\right)$, where $C_{i}$ are indicated by dashed lines, and $f$ is uniquely determined by the cuts.

For the definition of composition of morphisms and a proof why it is associative, please see [6]. The category $\mathcal{C} \mathcal{R} \mathcal{F}_{S}$ has several nice properties:

1. The empty forest $\emptyset$ is a null object in $\mathcal{C} \mathcal{R} \mathcal{F}_{S}$.

2. Disjoint union of forests equips $\mathcal{C} \mathcal{R} \mathcal{F}_{S}$ with a symmetric monoidal structure. We denote by $F_{1} \oplus F_{2}$ the disjoint union of the rooted forests $F_{1}$ and $F_{2}$ labeled by $S$, and refer to this as the direct sum.

3. Every morphism possesses a kernel and a cokernel.

4. For every admissible cut $C$ on a forest $F$, we have the short exact sequence

$$
\emptyset \rightarrow P_{C}(F) \stackrel{\left(C_{n u l l}, C, i d\right)}{\longrightarrow} F \stackrel{\left(C, C_{f u l l}, i d\right)}{\longrightarrow} R_{C}(F) \rightarrow \emptyset .
$$

The second property above allows us to define the Grothendieck group of $\mathcal{C} \mathcal{R} \mathcal{F}_{S}$ as

$$
K_{0}\left(\mathcal{C} \mathcal{R} \mathcal{F}_{S}\right):=\mathbb{Z}\left[\operatorname{Iso}\left(\mathcal{C R} \mathcal{F}_{S}\right] / \sim\right.
$$

i.e. the free abelian group generated by isomorphism classes of objects modulo the relation $\sim$, where $\sim$ is generated by differences $B-A-C$ for short exact sequences

$$
\emptyset \rightarrow A \rightarrow B \rightarrow C \rightarrow \emptyset .
$$

We denote by $[A]$ the class of $A \in \mathcal{C} \mathcal{R} \mathcal{F}_{S}$ in $K_{0}\left(\mathcal{C} \mathcal{R} \mathcal{F}_{S}\right)$. 
Lemma 2.1. $K_{0}\left(\mathcal{C} \mathcal{R} \mathcal{F}_{S}\right) \simeq \mathbb{Z}^{\oplus|S|}$

Proof. Let $\bullet_{s}$ denote the singleton rooted tree colored $s$. We observe that by repeated application of 2.1, any rooted forest $F$ is equivalent in $K_{0}\left(\mathcal{C R} \mathcal{F}_{S}\right)$ to a sum of such, coming from the vertices of $F$. To say this slightly differently, let $v(F, s)$ denote the number of vertices in $F$ of color $s \in S$, and let $\mathbb{Z}^{S}$ denote the free abelian group on the set $S$, with generators $e_{s}, s \in S$. Let

$$
\begin{aligned}
\Psi: \mathbb{Z}\left[\operatorname{Iso}\left(\mathcal{C} \mathcal{R} \mathcal{F}_{S}\right)\right] & \rightarrow \mathbb{Z}^{S} \\
\Psi(F) & =\sum_{s \in S} v(F, s) e_{s}
\end{aligned}
$$

The subgroup generated by the relations $\sim$ lies in the kernel of $\Psi$, so we get a well-defined group homomorphism

$$
\bar{\Psi}: K_{0}\left(\mathcal{C} \mathcal{R} \mathcal{F}_{S}\right) \rightarrow \mathbb{Z}^{S}
$$

Now, let

$$
\begin{aligned}
\Phi: \mathbb{Z}^{S} & \rightarrow K_{0}\left(\mathcal{C} \mathcal{R} \mathcal{F}_{S}\right) \\
\Phi\left(\sum_{s} a_{s} e_{s}\right) & =\sum_{s \in S} a_{s}\left[\bullet \bullet_{s}\right] .
\end{aligned}
$$

$\bar{\Psi}$ and $\Phi$ are easily seen to be inverse to each other.

We denote by $K_{0}^{+}\left(\mathcal{C} \mathcal{R} \mathcal{F}_{S}\right) \simeq \mathbb{N}^{|S|}$ the cone of effective classes in $K_{0}\left(\mathcal{C} \mathcal{R} \mathcal{F}_{S}\right)$.

\section{Ringel-Hall algebras}

We recall the definition of the Ringel-Hall algebra of $\mathcal{C} \mathcal{R} \mathcal{F}_{S}$ following [6]. For an introduction to Ringel-Hall algebras in the context of abelian categories, see [8]. We define the Ringel-Hall algebra of $\mathcal{C} \mathcal{R} \mathcal{F}_{S}$, denoted $\mathrm{H}_{\mathcal{C R F}_{S}}$, to be the $\mathbb{Q}$-vector space of finitely supported functions on isomorphism classes of $\mathcal{C} \mathcal{R} \mathcal{F}_{S}$. I.e.

$$
\mathrm{H}_{\mathcal{C R F}_{S}}:=\left\{f: \operatorname{Iso}\left(\mathcal{C R} \mathcal{F}_{S}\right) \rightarrow \mathbb{Q} \mid \# \operatorname{supp}(f)<\infty\right\}
$$

As a $\mathbb{Q}$-vector space it is spanned by the delta functions $\delta_{A}$, for $A \in \operatorname{Iso}\left(\mathcal{C R} \mathcal{F}_{S}\right)$. The algebra structure on $\mathrm{H}_{\mathcal{C R F}_{S}}$ is given by the convolution product:

$$
f \star g(M):=\sum_{A \subset M} f(A) g(M / A) .
$$

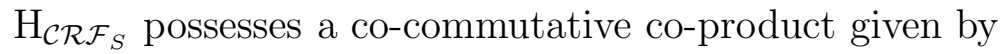

$$
\Delta(f)(M, N)=f(M \oplus N),
$$


as well as a natural $K_{0}^{+}\left(\mathcal{C} \mathcal{R} \mathcal{F}_{S}\right)$-grading in which $\delta_{A}$ has degree $[A] \in K_{0}^{+}\left(\mathcal{C} \mathcal{R} \mathcal{F}_{S}\right)$. The algebra and co-algebra structures are compatible, and $\mathrm{H}_{\mathcal{C R}_{\mathcal{F}}}$ is in fact a Hopf algebra (see [6]). It follows from 3.1 that

$$
\Delta\left(\delta_{A}\right)=\sum_{A^{\prime} \oplus A^{\prime \prime} \simeq A} \delta_{A^{\prime}} \otimes \delta_{A^{\prime \prime}}
$$

where the sum is taken over all distinct ways of writing $A$ as $A^{\prime} \oplus A^{\prime \prime}$.

\section{$4 \quad K_{0}^{+}\left(\mathcal{C} \mathcal{R} \mathcal{F}_{S}\right)$-graded noncommutative symmetric functions and homomorphisms}

Let $\mathrm{NC}_{\mathcal{C R F}_{S}}$ denote the free associative algebra on $K_{0}^{+}\left(\mathcal{C} \mathcal{R} \mathcal{F}_{S}\right)$, i.e. the free algebra generated by variables $X_{\alpha}$, for $\alpha \in K_{0}^{+}\left(\mathcal{C} \mathcal{R} \mathcal{F}_{S}\right)$. We give it the structure of a Hopf algebra through the coproduct

$$
\Delta\left(X_{\gamma}\right):=\sum_{\substack{\alpha+\beta=\gamma \\ \alpha, \beta \in K_{0}^{+}\left(\mathcal{C} \mathcal{R} \mathcal{F}_{S}\right)}} X_{\alpha} \otimes X_{\beta},
$$

and equip it with a $K_{0}^{+}\left(\mathcal{C} \mathcal{R} \mathcal{F}_{S}\right)$-grading by assigning $X_{\alpha}$ degree $\alpha$. This is a $K_{0}^{+}\left(\mathcal{C R} \mathcal{F}_{S}\right)$ graded version of the Hopf algebra of non-commutative symmetric functions (see [3]).

For $\alpha \in K_{0}^{+}\left(\mathcal{C} \mathcal{R} \mathcal{F}_{S}\right)$, let $\kappa_{\alpha}$ be the element of $\mathrm{H}_{\mathcal{C R F}_{S}}$ given by

$$
\kappa_{\alpha}:=\sum_{A \in \operatorname{Iso}(\mathcal{C}),[A]=\alpha} \delta_{A} .
$$

Example 4.1. Suppose that $S=\{a, b\}$. We then have $K_{0}\left(\mathcal{C R \mathcal { F }} \mathcal{F}_{S}\right) \simeq \mathbb{Z}^{2}$, and may identify the pair $(i, j) \in K_{0}^{+}\left(\mathcal{C R F}_{S}\right)$ as the class representing forests possessing $i$ vertices colored " $a$ " and $j$ colored " $b$ ". We have for instance

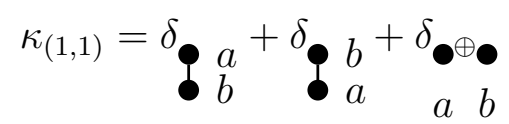

Theorem 1. The map $\rho: \mathrm{NC}_{\mathcal{C R}_{\mathcal{F}}} \rightarrow \mathrm{H}_{\mathcal{C R F}_{S}}$ determined by $\rho\left(X_{\alpha}\right)=\kappa_{\alpha}$ is a Hopf algebra homomorphism.

Proof. Since $\mathrm{NC}_{\mathcal{C R F}_{S}}$ is free as an algebra, we only need to check that the $\kappa_{\alpha}$ are compatible with the coproducts 4.1 , i.e. that

$$
\Delta\left(\kappa_{\gamma}\right)=\sum_{\substack{\alpha+\beta=\gamma \\ \alpha, \beta \in K_{0}^{+}\left(\mathcal{C} \mathcal{R F}_{S}\right)}} \kappa_{\alpha} \otimes \kappa_{\beta} .
$$


We have

$$
\begin{aligned}
\Delta\left(\kappa_{\gamma}\right) & =\sum_{\substack{A \in \operatorname{Iso}\left(\mathcal{C R} \mathcal{F}_{S}\right) \\
[A]=\gamma}} \Delta\left(\delta_{A}\right) \\
& =\sum_{\substack{A \in \operatorname{Iso}\left(\mathcal{C R} \mathcal{F}_{S}\right) \\
[A]=\gamma}} \sum_{A^{\prime} \oplus A^{\prime \prime} \simeq A} \delta_{A^{\prime}} \otimes \delta_{A^{\prime \prime}}
\end{aligned}
$$

The result now follows by observing that the term $\delta_{A^{\prime}} \otimes \delta_{A^{\prime \prime}}$ occurs exactly once in $\kappa_{\left[A^{\prime}\right]} \otimes$ $\kappa_{\left[A^{\prime \prime}\right]}$, which is an element of the right-hand side of 4.2 , since $\left[A^{\prime}\right]+\left[A^{\prime \prime}\right]=\gamma$.

\subsection{Connection to work of W. Zhao}

Let NC denote the "usual" Hopf algebra of non-commutative symmetric functions. I.e. $\mathrm{NC}$ is the free algebra on generators $Y_{n}, n \in \mathbb{N}$, with coproduct defined by

$$
\Delta\left(Y_{n}\right)=\sum_{i+j=n} Y_{i} \otimes Y_{j}
$$

(we adopt the convention that $Y_{0}=1$ ). Suppose that the labeling set $S$ is a subset of $\mathbb{N}$. We then have group homomorphism

$$
\begin{aligned}
V: K_{0}\left(\mathcal{C} \mathcal{R} \mathcal{F}_{S}\right) & \rightarrow \mathbb{N} \\
V\left(\sum a_{s} e_{s}\right) & :=\sum a_{s} s,
\end{aligned}
$$

which amounts to adding up the labels in a given forest. We can now define an algebra homomorphism

$$
\begin{aligned}
J_{S}: \mathrm{NC} & \rightarrow \operatorname{NC}_{\mathcal{C R \mathcal { F }}_{S}} \\
J_{S}\left(Y_{n}\right) & :=\sum_{\substack{\alpha \in K_{0}^{+}\left(\mathcal{C R} \mathcal{F}_{S}\right) \\
V(\alpha)=n}} X_{\alpha} .
\end{aligned}
$$

Lemma 4.1. $J_{S}$ is a Hopf algebra homomorphism

Proof. We only need to check the compatibility of the coproduct. We have

$$
\begin{aligned}
\Delta\left(J_{S}\left(Y_{n}\right)\right)= & \sum_{\substack{\alpha \in K_{0}^{+}\left(\mathcal{C R} \mathcal{F}_{S}\right) \\
V(\alpha)=n}} \Delta\left(X_{\alpha}\right) \\
= & \sum_{\substack{\alpha \in K_{0}^{+}\left(\mathcal{C} \mathcal{R} \mathcal{F}_{S}\right) \\
V(\alpha)=n}} \sum_{\gamma_{1}+\gamma_{2}=\alpha} X_{\gamma_{1}} \otimes X_{\gamma_{2}} \\
= & \sum_{\substack{\gamma, \gamma^{\prime} \\
V(\gamma)+V\left(\gamma^{\prime}\right)=n}} X_{\gamma} \otimes X_{\gamma^{\prime}} \\
= & J_{S}\left(\Delta\left(Y_{n}\right)\right) .
\end{aligned}
$$


Composing $\rho$ and $J_{S}$, we obtain a Hopf algebra homomorphism

$$
\begin{aligned}
\rho \circ J_{S}: \mathrm{NC} & \rightarrow \mathrm{H}_{\mathcal{C R \mathcal { F }}_{S}} \\
\rho \circ J_{S}\left(Y_{n}\right) & =\sum_{\substack{A \in \operatorname{Iso}\left(\mathcal{C R} \mathcal{F}_{S}\right) \\
V([A])=n}} \delta_{A}
\end{aligned}
$$

which was considered in [9].

\section{The transpose of $\rho$}

The graded dual of the Hopf algebra $\mathrm{NC}_{\mathcal{C R F}_{S}}$ is a $K_{0}^{+}(\mathcal{C})$-graded version of the Hopf algebra of quasi-symmetric functions (see [1]), which we proceed to describe. Let QSym $\mathcal{C R F}_{S}$ denote the $\mathbb{Q}$-vector space spanned by the symbols $Z\left(\alpha_{1}, \alpha_{2}, \ldots, \alpha_{k}\right)$, for $k \in \mathbb{N}$, and $\alpha_{i} \in K_{0}^{+}\left(\mathcal{C R F}_{S}\right)$. We make $\mathrm{QSym}_{\mathcal{C R}_{\mathcal{F}}}$ into a co-algebra via the coproduct

$$
\begin{aligned}
\Delta\left(Z\left(\alpha_{1}, \ldots, \alpha_{k}\right)\right) & =1 \otimes Z\left(\alpha_{1}, \ldots, \alpha_{k}\right) \\
& +\sum_{i=1}^{k-1} Z\left(\alpha_{1}, \ldots, \alpha_{i}\right) \otimes Z\left(\alpha_{i+1}, \ldots, \alpha_{k}\right)+Z\left(\alpha_{1}, \ldots, \alpha_{k}\right) \otimes 1
\end{aligned}
$$

The algebra structure on $\mathrm{QSym}_{\mathcal{C R F}_{S}}$ is given by the quasi-shuffle product, as follows. Given $Z\left(\alpha_{1}, \ldots, \alpha_{k}\right)$ and $Z\left(\beta_{1}, \ldots, \beta_{l}\right)$, their product is determined by:

1. Inserting zeros into the sequences $\alpha_{1}, \ldots, \alpha_{k}$ and $\beta_{1}, \ldots, \beta_{l}$ to obtain two sequences $\nu_{1}, \ldots, \nu_{p}$ and $\mu_{1}, \ldots, \mu_{p}$ of the same length, subject to the condition that for no $i$ do we have $\nu_{i}=\mu_{i}=0$.

2. For each such pair $\nu_{1}, \ldots \nu_{p}$, and $\mu_{1}, \ldots, \mu_{p}$, writing $Z\left(\nu_{1}+\mu_{1}, \ldots, \nu_{p}+\mu_{p}\right)$.

3. Summing over all possible such pairs of sequences $\left\{\nu_{1}, \ldots, \nu_{p}\right\},\left\{\mu_{1}, \ldots, \mu_{p}\right\}$.

Example 5.1. We have

$$
\begin{aligned}
Z\left(\alpha_{1}\right) Z\left(\beta_{1}, \beta_{2}\right) & =Z\left(\alpha_{1}+\beta_{1}, \beta_{2}\right)+Z\left(\beta_{1}, \alpha_{1}+\beta_{2}\right)+Z\left(\beta_{1}, \beta_{2}, \alpha_{1}\right) \\
& +Z\left(\beta_{1}, \alpha_{1}, \beta_{2}\right)+Z\left(\alpha_{1}, \beta_{1}, \beta_{2}\right) .
\end{aligned}
$$

One checks readily that the two structures are compatible, and that they respect the $K_{0}^{+}\left(\mathcal{C} \mathcal{R} \mathcal{F}_{S}\right)$-grading determined by

$$
\operatorname{deg}\left(Z\left(\alpha_{1}, \ldots, \alpha_{k}\right)\right)=\alpha_{1}+\ldots+\alpha_{k}
$$

The pairing

$$
\langle,\rangle: \operatorname{QSym}_{\mathcal{C R F}_{S}} \times \mathrm{NC}_{\mathcal{C R}_{\mathcal{F}}} \rightarrow \mathbb{Q}
$$


determined by

$$
\left\langle Z\left(\alpha_{1}, \ldots, \alpha_{n}\right), X_{\beta_{1}} \ldots X_{\beta_{m}}\right\rangle:=\delta_{m, n} \delta_{\alpha_{1}, \beta_{1}} \ldots \delta_{\alpha_{n}} \delta_{\beta_{m}}
$$

makes $\mathrm{QSym}_{\mathcal{C R F}_{S}}$ and $\mathrm{NC}_{\mathcal{C R F}_{S}}$ into a dual pair of $K_{0}^{+}\left(\mathcal{C} \mathcal{R} \mathcal{F}_{S}\right)$-graded Hopf algebras. I.e.

$$
\begin{aligned}
\langle a \otimes b, \Delta(v)\rangle & =\langle a b, v\rangle \\
\langle\Delta(a), v \otimes w\rangle & =\langle a, v w\rangle .
\end{aligned}
$$

This implies that $\mathrm{QSym}_{\mathcal{C R F}_{S}}$ is isomorphic to the graded dual of $\mathrm{NC}_{\mathcal{C R F}_{S}}$. Passing to graded duals, and taking the transpose of the homomorphism $\rho$, we obtain a Hopf algebra homomorphism

$$
\rho^{t}: \mathrm{H}_{\mathcal{C R F}_{S}}^{*} \rightarrow \operatorname{QSym}_{\mathcal{C R F}_{S}} .
$$

As shown in [6], $\mathrm{H}_{\mathcal{C} \mathcal{R} \mathcal{F}_{S}}^{*}$ is isomorphic to the Connes-Kreimer Hopf algebra on colored trees (see [5]).

We proceed to describe $\rho^{t}$. Let $\left\{W_{A}, A \in \operatorname{Iso}\left(\mathcal{C} \mathcal{R} \mathcal{F}_{S}\right)\right\}$ be the basis of $\mathrm{H}_{\mathcal{C} \mathcal{R} \mathcal{F}_{S}}^{*}$ dual to the basis $\left\{\delta_{A}\right\}$ of $\mathrm{H}_{\mathcal{C R}_{\mathcal{F}}}$.

\section{Theorem 2.}

$$
\rho^{t}\left(W_{A}\right)=\sum_{k} \sum_{V_{1} \subset \ldots \subset V_{k}=A} Z\left(\left[V_{1}\right],\left[V_{2} / V_{1}\right], \ldots,\left[V_{k} / V_{k-1}\right]\right)
$$

where the inner sum is over distinct $k$-step flags

$$
V_{1} \subset V_{2} \subset \ldots \subset V_{k}=A, V_{i} \in \operatorname{Iso}\left(\mathcal{C} \mathcal{R F}_{S}\right)
$$

Proof. We have

$$
\rho^{t}\left(W_{A}\right)\left(X_{\alpha_{1}} \ldots X_{\alpha_{k}}\right)=N\left(A ; \alpha_{1}, \ldots, \alpha_{k}\right),
$$

where $N\left(A ; \alpha_{1}, \ldots, \alpha_{k}\right)$ is the coefficient of $\delta_{A}$ in the product $\kappa_{\alpha_{1}} \kappa_{\alpha_{2}} \ldots \kappa_{\alpha_{k}}$. It follows from the definition of the multiplication in the Ringel-Hall algebra that this is exactly the number of flags

$$
V_{1} \subset V_{2} \ldots \subset V_{k}
$$

where $\left[V_{1}\right]=\alpha_{1},\left[V_{2} / V_{1}\right]=\alpha_{2}, \ldots,\left[V_{k} / V_{k-1}\right]=\alpha_{k}$.

Example 5.2. Let $S=\{a, b\}$ as in example 4.1. Using the notation introduced there, we have

$$
\begin{aligned}
& \rho^{t}(W \underset{\sigma}{W})=Z((2,1))+Z((0,1),(2,0))+Z((1,0),(1,1)) \\
& \text { b a } \\
& +Z((1,1),(1,0))+Z((1,0),(0,1),(1,0))+Z((0,1),(1,0),(1,0)) .
\end{aligned}
$$




\section{References}

[1] Cartier, P., A primer of Hopf algebras. Frontiers in number theory, physics, and geometry. II, 537-615, Springer, Berlin, 2007.

[2] Connes, A. and Kreimer, D., Hopf algebras, renormalization, and noncommutative geometry. Comm. Math. Phys. 199 203-242 (1998).

[3] Gelfand I; Krob D; Lascoux A; Leclerc B; Retakh V; Thibon J-Y. Noncommutative symmetric functions. Adv. Math. 112 (1995), no. 2, 218-348.

[4] Joyce, D., Configurations in abelian categories. II. Ringel-Hall algebras. Adv. Math. 210 no. 2, 635-706 (2007).

[5] Kreimer, D., On the Hopf algebra structure of perturbative quantum field theory. Adv. Theor. Math. Phys. 2 303-334 (1998).

[6] Kremnizer K. and Szczesny M., Feynman graphs, rooted trees, and Ringel-Hall algebras. Comm. Math. Phys. 289 (2009), no. 2 561-577.

[7] Ringel, C., Hall algebras, Topics in algebra, Part 1 (Warsaw, 1988), 433447, Banach Center Publ., 26, Part 1, PWN, Warsaw, (1990).

[8] Schiffmann, O., Lectures on Hall algebras. Preprint math.RT/0611617.

[9] Zhao, W., A noncommutative symmetric system over the Grossman-Larson Hopf algebra of labeled rooted trees. J. Algebraic Combin . 28 (2008), no. 2, 235-260. 